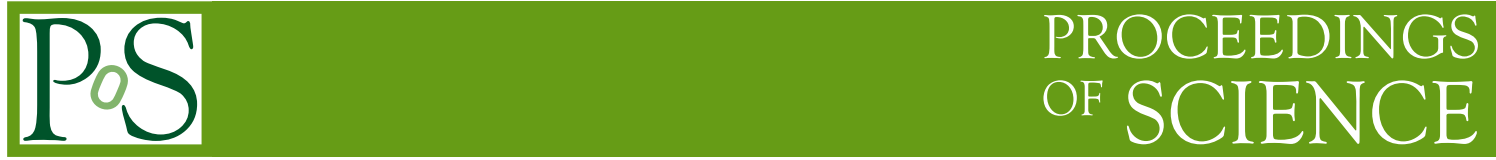

\title{
Comments on staggered fermions / Panel discussion
}

\author{
Michael Creutz ${ }^{* \dagger}$ \\ Brookhaven National Laboratory \\ E-mail: creutz@bnl.gov
}

The rooting procedure commonly used with staggered fermions does not correctly treat nonperturbative effects associated with gauge field topology. In practice these effects are small for the physics of flavor non-singlet particles. However large uncontrolled systematic errors are expected for flavor singlet issues, such as the mass of the eta prime meson. While the relative speed of the algorithm in large scale simulations may justify its use, the method is an approximation and should not be promoted as a first principles approach to the strong interactions.

8th Conference Quark Confinement and the Hadron Spectrum

September 1-6, 2008

Mainz. Germany

\section{* Speaker.}

$\dagger$ This manuscript has been authored under contract number DE-AC02-98CH10886 with the U.S. Department of Energy. Accordingly, the U.S. Government retains a non-exclusive, royalty-free license to publish or reproduce the published form of this contribution, or allow others to do so, for U.S. Government purposes. 
Within the lattice gauge community there currently exists a rather bitter ongoing controversy over whether one should be using the rooted staggered fermion algorithm for large scale simulations of QCD. While the approach has been shown to be a good approximation for many quantities, it mistreats anomaly effects and thus is not a first principles approach to the strong interactions. Here I summarize the issues for the broader audience at this conference.

As all here are familiar, chiral symmetry plays a key role in our understanding of the strong interactions. From a conceptual point of view, pions are most elegantly described as waves propagating on a quark condensate, $\langle\bar{\psi} \psi\rangle$. And from a practical point of view, chiral extrapolations are an essential tool for the lattice gauge theorist to extract predictions from simulations with quark masses heavier than their physical values.

The subject of chiral symmetry is intricately entwined with quantum anomalies. As is well known, these remove the classical $U(1)$ chiral symmetry of the theory. For $N_{f}$ massless quark flavors, the surviving chiral symmetry is $S U\left(N_{f}\right)_{L} \times S U\left(N_{f}\right)_{R}$. Since $S U(1)$ is trivial, a useful chiral symmetry requires at least two flavors. Indeed, an exact chiral symmetry for two flavors is possible on the lattice using minimally doubled actions [1], but that is not the subject of this session.

Ignoring the anomaly in lattice gauge theory frequently leads to what are known as doublers. For example, the most naive fermion action has quarks hopping from site to site picking up gamma matrix factors. That approach maintains chiral symmetry, but on further analysis actually describes sixteen fermions in the continuum limit. The formulation possesses an exact $U(4) \times U(4)$ chiral symmetry [2]. Dividing out that symmetry gives rise to the staggered fermion approach. This leaves behind four remaining species, often called tastes, while maintaining one exact chiral symmetry $[3,4,5]$. The alternative Wilson fermion approach does succeed in eliminating all doubling, but at the expense of breaking any remnants of chiral symmetry at finite lattice spacing [6]. Other techniques, such as perfect actions [7], domain wall quarks [8], or the overlap operator [9], do maintain a form of chiral symmetry for any $N_{f}$, although these all involve interactions over a range of sites and thus are extremely computationally intensive. Furthermore, with these actions the anomaly is often somewhat hidden; for example, the overlap formalism introduces two different chiral matrices $\gamma_{5}$ and $\hat{\gamma}_{5}$, with the latter being non-local, gauge field dependent, and having a trace related to the winding number of the background gauge field. The minimally doubled actions do maintain a strict locality along with one exact chiral symmetry, but suffer from potential lattice distortions that should be tuned at finite lattice spacing.

The issue at the heart of the recent controversies over staggered fermions is the process referred to as rooting. In an attempt to remove the fourfold multiplicity of the staggered approach, simulations are done with the determinant of the staggered operator replaced with its fourth root. In a perturbative expansion this multiplies each fermion loop by $1 / 4$, correcting for the extra multiplicity of the four tastes. Thus rooting is a valid procedure in perturbation theory. But all symmetries of the fermion determinant are kept by its fourth root, and the one flavor theory has no chiral symmetry. Thus something must go wrong at a non-perturbative level.

At last year's lattice meeting in Regensburg I went into some detail as to how this rooting procedure fails. Those that are interested in the specifics can refer to Ref. [10]. Further discussion can be found in Refs. [11] and [12]. Here I discuss the qualitative essence of the problem, which is that the rooting procedure does not treat instanton effects properly. 


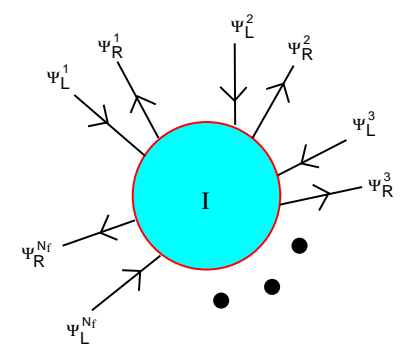

Figure 1: A topologically non-trivial gauge field generates an effective interaction between all fermion species, each of which flips its spin.

Whenever the gauge fields are topologically non-trivial, the continuum Dirac operator develops real eigenvectors, and these appear for every flavor and taste. At zero mass the corresponding eigenvalues go to zero. Depending on the action, lattice artifacts can mix these modes and make them not exactly real, but near the continuum limit they should exist as approximately real eigenvalues. Furthermore, in the continuum these modes become chiral, i.e. the eigenvectors are also eigenstates of $\gamma_{5}$, with the eigenvalue depending on the winding number of the gauge field. The physical effect of these modes is an effective coupling of all species whenever an instanton is present [13]. This is sketched in Fig. (1). Whether or not the gauge configurations are obtained with rooting, this coupling involves every taste at once. The net effect eliminates the possibility of considering the tastes as independent, and the factorization crucial to the perturbative argument fails. In particular, the resulting coupling between tastes should not be present in the target theory with a reduced number of species.

Unfortunately numerous misleading statements continue to be propagated about this problem. To begin with, it has nothing to do with a breaking of the symmetry between the tastes. The unwanted coupling takes the form of a determinant which preserves this symmetry. Second, the issue is not associated with taking the fermion mass to zero. The effective coupling is present at finite mass, and indeed is enhanced with the mass since instantons are suppressed in the chiral limit. Finally, and most important, the problematic coupling does not go away in the continuum limit. The issues occur at the typical instanton scale, which is set by $\Lambda_{\mathrm{qcd}}$. Simple symmetry arguments forbid a reduction to the desired form of the instanton induced interaction in the target theory.

To see this more explicitly, note that with $N_{f}$ physical flavors, in addition to the usual flavored chiral symmetries, massless continuum QCD has a $Z_{N_{f}}$ discrete chiral symmetry [14] under

$$
\psi_{L} \rightarrow e^{2 \pi i / N_{f}} \psi_{L}
$$

Here I have chosen a left handed chiral rotation; equivalently one could work with $\psi_{R}$. This symmetry can be seen either directly from the 't Hooft vertex, which receives a factor from each species, or from the conventional chiral Lagrangian approach because $e^{2 \pi i / N}$ is an element of the flavored chiral symmetry group $S U(N)$. In terms of the singlet composite fields $\sigma \sim \bar{\psi} \psi$ and $\eta^{\prime} \sim i \bar{\psi} \gamma_{5} \psi$ (sum over flavors implied), the effective potential of the theory is symmetric under

$$
\begin{gathered}
\sigma \rightarrow \cos \left(2 \pi / N_{f}\right) \sigma-\sin \left(2 \pi / N_{f}\right) \eta^{\prime} \\
\eta^{\prime} \rightarrow \sin \left(2 \pi / N_{f}\right) \sigma+\cos \left(2 \pi / N_{f}\right) \eta^{\prime} .
\end{gathered}
$$



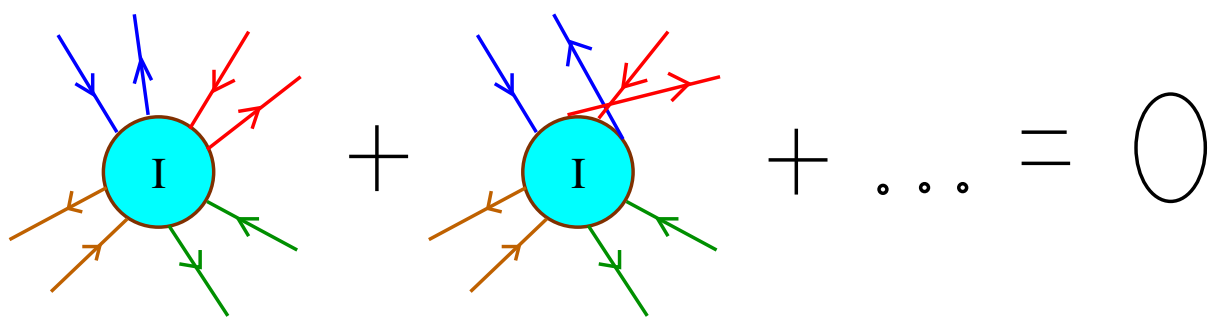

Figure 2: Were one to root four copies of a single fermion, Pauli statistics require including exchange diagrams that cancel the analogue of the coupling between the four tastes of staggered fermions.

Note that because the anomaly generates an $\eta^{\prime}$ mass, the theory must not be symmetric under $U(1)$ rotations of the above form with angles less than $2 \pi / N_{f}$.

Now the staggered fermion operator has $4 N_{f}$ effective flavors. This means that near the continuum limit the action possesses a $Z_{4 N_{f}}$ symmetry. This is true for any gauge configuration, independent of whether it is obtained by rooting or not. The excess symmetry forbids a reduction to the desired $Z_{N_{f}}$ symmetry for the rooted theory.

It has been argued that because of the trivial mathematical identity $\left(|D|^{4}\right)^{1 / 4} \equiv|D|$, rooting four copies of a single fermion should work, invalidating the above discussion. This misses the crucial point that staggered fermions are not four copies of one fermion. Indeed, as discussed in some detail in [10], the different tastes have different chiralities. When a topological defect is present, two tastes have a left handed zero mode and two are right handed. Rooting effectively averages over physically inequivalent states.

More generally, the staggered propagator gives rise to four poles representing the four inequivalent fermions. Since the staggered Dirac operator is normal, the multiple eigenvectors associated with zero modes are exactly orthogonal. In contrast, for the correct one flavor theory there is only one zero mode. With a single state, Pauli statistics must be taken into account and will cancel any analogous contributions from multiple copies of the single fermion, as sketched in Fig. (2).

So rooting is wrong, but could it be a good approximation? The answer here appears to be yes, based on the many good past results. This can be partially understood from the fact that virtual quark loops are a relatively small correction to the valence approximation. Also, since the problems involve all flavors and tastes at once, the issues don't arise until an effective $N_{f}$ loop order. Nevertheless, results from rooting that involve singlet processes, where topological effects are important, are extremely suspect.

To conclude, the basic question is "Does expediency justify rooted staggered quarks?" Obviously I don't think so, but some others in the lattice gauge community feel differently. I summarize in Table 1 the main arguments for and against using this algorithm. I stress, however, that if rooting is used, it is necessary to admit that it is an approximation involving uncontrolled systematic errors when instanton physics is important. 


\begin{tabular}{|c|c|}
\hline Pro & Con \\
\hline $\begin{array}{l}\text { A good approximation } \\
\text { - good for flavor physics }\end{array}$ & $\begin{array}{l}\text { But not first principles QCD } \\
\text { - incorrect singlet physics }\end{array}$ \\
\hline $\begin{array}{l}\text { Fast simulations } \\
\text { - thermo needs high statistics }\end{array}$ & $\begin{array}{l}\text { Wilson fermions not far behind [15] } \\
\text { - twisted catching up }\end{array}$ \\
\hline $\begin{array}{l}\text { Exact chiral symmetry } \\
\text { - simple chiral extrapolations }\end{array}$ & $\begin{array}{l}\text { Even when wrong; i.e. } N_{f}=1 \\
\text { - valid chiral algorithms exist }\end{array}$ \\
\hline Much faster than D & nally doubled actions \\
\hline Big lattices freely available & You get what you pay for \\
\hline A lot already invested & Don't throw good money after bad \\
\hline
\end{tabular}

Table 1: Arguments pro and con for investigating lattice gauge theory using rooted staggered quarks.

\section{References}

[1] L. H. Karsten, Phys. Lett. B 104, 315 (1981); F. Wilczek, Phys. Rev. Lett. 59, 2397 (1987); M. Creutz, JHEP 0804, 017 (2008) [arXiv:0712.1201 [hep-lat]]; A. Borici, arXiv:0712.4401 [hep-lat].

[2] L. H. Karsten and J. Smit, Nucl. Phys. B 183, 103 (1981).

[3] J. B. Kogut and L. Susskind, Phys. Rev. D 11, 395 (1975).

[4] L. Susskind, Phys. Rev. D 16, 3031 (1977).

[5] H. S. Sharatchandra, H. J. Thun and P. Weisz, Nucl. Phys. B 192, 205 (1981).

[6] K. G. Wilson, New Phenomena In Subnuclear Physics. Part A. Proceedings of the First Half of the 1975 International School of Subnuclear Physics, Erice, Sicily, July 11 - August 1, 1975, ed.

A. Zichichi, Plenum Press, New York, 1977, p. 69, CLNS-321

[7] P. Hasenfratz and F. Niedermayer, Nucl. Phys. B 414 (1994) 785 [arXiv:hep-lat/9308004];

W. Bietenholz and U. J. Wiese, Nucl. Phys. B 464, 319 (1996) [arXiv:hep-lat/9510026].

[8] D. B. Kaplan, Phys. Lett. B 288 (1992) 342 [arXiv:hep-lat/9206013]; V. Furman and Y. Shamir, Nucl. Phys. B 439, 54 (1995) [arXiv:hep-lat/9405004].

[9] H. Neuberger, Phys. Lett. B 417, 141 (1998) [arXiv:hep-lat/9707022].

[10] M. Creutz, PoS LATTICE2007, 007 (2007) [arXiv:0708.1295 [hep-lat]].

[11] M. Creutz, Phys. Lett. B 649 (2007) 230 [arXiv:hep-lat/0701018];

[12] M. Creutz, Annals Phys. 323, 2349 (2008) [arXiv:0711.2640 [hep-ph]].

[13] G. 't Hooft, Phys. Rev. Lett. 37 (1976) 8; G. 't Hooft, Phys. Rev. D 14, 3432 (1976) [Erratum-ibid. D 18, 2199 (1978)].

[14] M. Creutz, Phys. Rev. D 52, 2951 (1995) [arXiv:hep-th/9505112].

[15] L. Del Debbio, L. Giusti, M. Luscher, R. Petronzio and N. Tantalo, JHEP 0702, 056 (2007) [arXiv:hep-lat/0610059]. 